\title{
Radio and Gamma-ray emission in nearby BL Lacs
}

\author{
G. Giovannini ${ }^{1,2}$, E. Liuzzo ${ }^{2}$, B. Boccardi ${ }^{3}$, and M. Giroletti ${ }^{2}$ \\ ${ }^{1}$ Department of Physics and Astronomy, via Ranzani 1, 40123 Bologna, Italy \\ email: ggiovann@ira.inaf.it \\ ${ }^{2}$ Radioastronomy Institute/INAF, via Gobetti 101, 40129 Bologna, Italy \\ ${ }^{3}$ Max Planck Institute for Radioastronomy, Auf dem Hügel 6953121 Bonn, Germany.
}

\begin{abstract}
The advent of Fermi is changing our understanding on the radio and $\gamma$-ray emission in active galactic nuclei. Unlike pre-Fermi ideas, BL Lac objects are found to be the most abundant emitters in the $\gamma$-ray band. However, since they are relatively weak radio sources, most of their parsec-scale structure and their multifrequency properties are poorly understood and/or have not been investigated in a systematic fashion. Here we are analyzing the radio and $\gamma$-ray emission properties of a sample of 42 BL Lacs selected with no constraint on their radio and $\gamma$-ray emission. Thanks to new Very Long Baseline Array observations at 8 and 15 $\mathrm{GHz}$ for the whole sample, we discuss their parsec-scale structure. Parsec-scale radio emission is observed in the majority of the sources at both frequencies. The comparison between our results in radio and gamma-ray bands points out the presence of a large number of faint BL Lacs showing "non-classical" properties such as low source compactness, low core dominance, no gamma-ray emission.
\end{abstract}

Keywords. BL Lacs, VLBI

\section{Introduction}

Thanks to the advent of the Fermi mission, studies of a large number of $\gamma$-ray sources have become possible. The Large Area Telescope (LAT, Atwood et al. 2009) has shown that the BL Lacs and not the FSRQs are now the most common $\gamma$-ray emitters (1LAC, Abdo et al. 2010; 2LAC, Ackermann et al. 2011): in the Clean 2 LAC Sample, 395 are BL Lac objects, 310 FSRQs, 157 sources of unknown type, and 22 other AGNs (Ackermann et al. 2011). Big questions nevertheless remain open, such as the origin and location of $\gamma$-rays, the distance of the main energy dissipation site from the nucleus, and the relation between the $\gamma$-ray and radio emission.

BL Lac objects belong to the blazar population showing a high core dominance, high degrees of variability and polarization, and one-sided Doppler beamed parsec-scale jets. The high-energy characteristics of BL Lacs can be investigated thanks to new Fermi results, but to understand the origin and the nuclear properties of these sources more information at different frequencies are necessary. Very Long Baseline Interferometer (VLBI) campaigns are one of the most incisive observational tools for addressing the questions opened by the Fermi results. However previous VLBI surveys have looked at the parcsec scale properties of the brightest BL Lac objects, while the majority of the BL Lac population is below their flux density limits (Wu et al. 2007; Giroletti et al. 2004; Giroletti et al. 2006; Rector et al. 2003; Cassaro et al. 2002). In fact, many LAT BL Lacs are high-frequency-synchrotron-peaked (HSP) sources, discovered at X-rays and generally they are faint radio sources, seldom studied with VLBI. To gain a deeper understanding 
of BL Lac properties, we selected a sample of low-redshift BL Lacs sources with no selection limits on their radio flux density or high-frequency emission.

Main results have been published in Liuzzo et al. (2013), here we shortly review main results and discuss in more detail the core dominance.

Throughout this paper, we assume $\mathrm{H}_{0}=0.71 \mathrm{~km} \mathrm{~s}^{-1} \mathrm{Mpc}^{-1}, \Omega_{m}=0.27$, and $\Omega_{\text {lambda }}=$ 0.73. We define radio spectral index $\alpha$ such that the flux density $\mathrm{S}(\nu) \propto \nu^{-\alpha}$.

\section{The sample}

We selected our sample of BL Lacs from the Roma BZCat Catalog of the known blazars (Massaro et al. 2009) with only two constraints:

- a measured redshift $\mathrm{z}<0.2$,

- BL Lacs located within the sky area covered by the Sloan Digital Sky Survey (SDSS, Abazajian et al. 2009).

These two selection criteria allow us 1) to investigate the least powerful sources, such as the weak population of HSP BL Lacs, with a good linear resolution $(1 \mathrm{pc} \sim 0.5$ mas at $\mathrm{z}=0.1) ; 2$ ) a good characterization not only of the optical properties, but also of their extended radio characteristics since the Faint Images of the Radio Sky at TwentyCentimeters (FIRST) cover the same SDSS field. The total number of BL Lacs in our sample is 42 . Our sample is representative of a population that is the majority of the whole BL Lacs, but it is not explored by the most complete previous VLBI surveys.

\section{Results}

\subsection{Detection rate}

In our sample, 27/42 (64\%) objects show parsec-scale radio emission. In particular, 24 $\mathrm{BL}$ Lac objects are revealed at both frequencies, while two are detected only at $8 \mathrm{GHz}$ and one only at $15 \mathrm{GHz}$.

Point-like morphologies are present in $8 / 27(31 \%)$ of the detected sources at $8 \mathrm{GHz}$, and in 14/26 (54\%) of the revealed targets at $15 \mathrm{GHz}$ (see Liuzzo et al. 2013). The other detected sources show a one-sided structure.

\subsection{The source radio structures}

We evaluated, for all objects, the source compactness (SC) value defined as the ratio of the $8 \mathrm{GHz}$ VLBA and the NVSS total powers. In Fig. 1-left, we plot the SC distribution for our sample. We show SC values for both detected and non-detected sources, assuming for the latter upper flux density limits. Most of our sources show a parsec-scale flux density that is significantly lower than expected from the kiloparsec-scale flux densities estimated from the NVSS and/or FIRST radio surveys. In most cases, this difference cannot be due to the radio spectrum or to variability, and it suggests the presence of a subkiloparsec radio structure lost in VLBA data because of the lack of short baselines.

Moreover, we estimated the core dominance of each BL Lac of the sample as following (see also Liuzzo et al. 2009): given the existence of a general correlation between the core (at $5 \mathrm{GHz}$ ) and total radio power (at $408 \mathrm{MHz}$ ):

$\log \mathrm{P}_{c}=0.62 \log \mathrm{P}_{\text {tot }}+7.6$ (Giovannini et al. 1994)

we can derive the expected core radio power from the unboosted total radio power at low frequency. This estimated radio power corresponds to a source orientation $\theta=60^{\circ}$. The Core Dominance (CD) is the ratio between the observed core radio power and the estimated core radio power according to the previous correlation from the unbeamed total radio power at low frequency. 

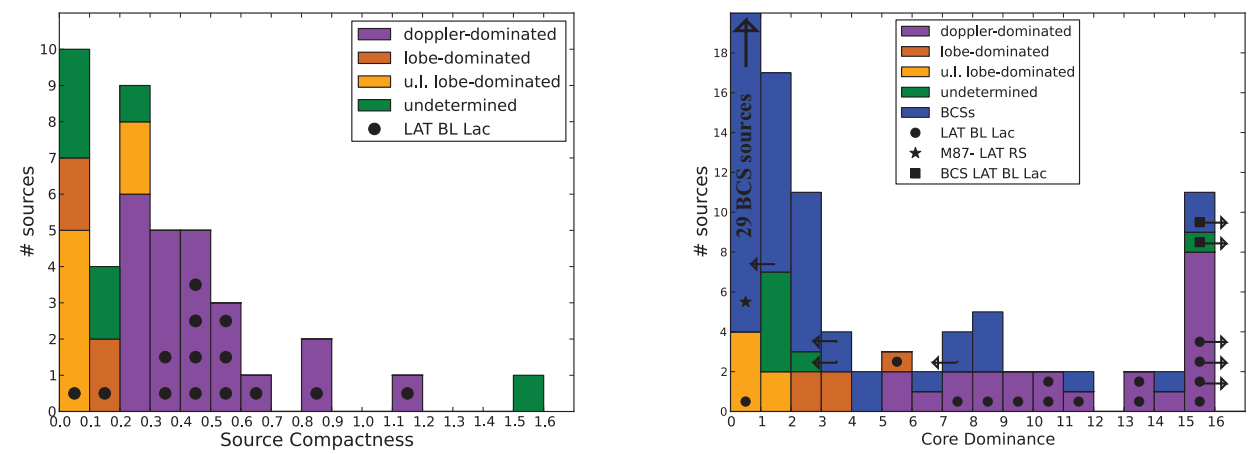

Figure 1. Source compactness (SC, on the left) and core dominance (CD, on the right) distributions for the present sample of BL Lacs.

Since in most of our sample a measure of the total radio power at low frequency is unavailable, we estimated the total radio power at $408 \mathrm{MHz}$ extrapolating the NVSS total radio power using an average spectral index $=0.7$. For the core radio power , we used the radio power at $8 \mathrm{GHz}$.

In Fig. 1-right, we show the CD distribution for our sample. We added also the CD of radio sources (radio galaxies and 2 BL Lacs) from the Bologna Complete Sample (BCS, see Liuzzo et al. 2009) for a comparison. As expected, the distribution of BL Lac sources peaks at higher core dominance according to unified models.

\section{Discussion}

\subsection{The source radio structures}

Looking at the derived values of source compactness and core dominance, together with the source morphology and radio spectrum, the BL Lac objects of our sample could be divided as follows:

- Doppler dominated (DD) sources: Among the 42 BL Lacs, we have 23 sources in which the emission is dominated by the central core. They are characterized by a CD always larger than 5.5 suggesting that they are relativistic Doppler boosted objects. We note that a CD larger than 5.5 implies a jet velocity $>0.7 \mathrm{c}$ and an orientation angle smaller than $35^{\circ}$ with respect to the line of sight.

Most of these sources display a core dominant structure and a one-sided jet, confirming the importance of relativistic effects. The SC is generally high, even if in some case the missing flux density is important, suggesting the presence of a structure more complex than the detected one-sided jet. For comparison, we note that radio galaxies of the BCS (Liuzzo et al. 2009) show in most cases a CD $<4$ and in many cases $<1$.

- Lobe dominated (LD) sources: In 11 cases, the VLBI total flux densities are a small fraction of the kiloparsec-scale ones, with $\mathrm{CD}<5.5$. This group includes both undetected and detected sources in our VLBA maps. In a few sources, the radio spectrum is quite steep, suggesting that they are dominated by an extended steep spectrum structure despite their classification as BL Lac objects.

- Undetermined (U) objects: There are eight sources that are not detected in our VLBA images. Their arcsec flux density is low, therefore we cannot establish whether the lack of the detection is due to the absence of a compact core or to a sensitivity problem. Deeper observations will be needed to investigate their parsec-scale properties. 


\subsection{Gamma ray properties}

Among BL Lacs in our sample 14/42 show high energy emission in the Second LAT AGN catalog. We will call them LAT BL Lacs. Among these LAT BL Lacs, two are classified as LD objects, the others as DD objects. The LD BL Lac J1534+3715 is the only LAT sources not detected by our VLBA observations Comparing their parsec scale properties, LAT BL Lacs are more luminous on mas scale respect to the non-LAT objects. In particular, all LAT BL Lacs with $\log \mathrm{P}_{V L B A, 8 G H z} \geqslant 24.6\left(\mathrm{~W} \mathrm{~Hz}^{-1}\right)$ present resolved morphologies both at 8 and $15 \mathrm{GHz}$. On the other hand, objects with $\log \mathrm{P}_{V L B A, 8 G H z} \leqslant$ $23.6\left(\mathrm{~W} \mathrm{~Hz}^{-1}\right)$ do not show $\gamma$-ray emission, as sources with core dominance lower than 5.5. All these characteristics confirm the idea that LAT BL Lacs are dominated by Doppler boosting effects.

\section{Conclusions}

1) Studying a sample of BL Lacs with no selection constrain on the radio and high energy flux we find a dual BL Lac population: DD sources where the presence of relativistic jets is evident from radio images; LD BL Lacs, where radio images do not show any evidence of the presence of relativistic jets with high Doppler factor.

2) Among sources detected by the Fermi satellite (2LAC) in our sample, 12 are DD and only 2 are LD objects, confirming the strong correlation between radio and high-energy properties.

3) In all low power BL Lacs the comparison between the correlated VLBA flux density and the NVSS flux density suggests the presence of a sub-kpc scale structure. This is more evident in sources not detected by Fermi and in faint sources.

4) The nature and properties of LD BL Lacs it is not yet clear. A connection with Core Galaxies studied e.g. by Balmaverde and Capetti, (2006) could be possible. We are investigating this point with new eMERLIN observations (obtained) and deeper VLBI observation (EVN submitted).

\section{References}

Abazajian, K. N., Adelman-McCarthy, J. K., Agüeros, M. A., et al. 2009 ApJS, 182, 543

Abdo, A. A., Ackermann, M., Ajello, M., et al. 2009 ApJ, 697, 1071

Ackermann, M., Ajello, M., Allafort, A., et al. 2011 ApJ, 743, 171

Atwood, W. B., Abdo, A. A., Ackermann, M., et al. 2009, ApJ, 697, 1071

Balmaverde, B., Capetti, A. 2006 A $\mathscr{S} A$ 447, 97

Cassaro, P., Stanghellini, C., Dallacasa, D., Bondi, M., \& Zappalà, R. A. 2002 A\&A 381, 378

Giovannini, G., Feretti, L., Venturi, T., et al. 1994 ApJ 435, 116

Giroletti, M., Giovannini, G., Taylor, G. B., \& Falomo, R. 2004 ApJ 613, 752

Giroletti, M., Giovannini, G., Taylor, G. B., \& Falomo, R. 2006 ApJ 646, 801

Liuzzo, E., Giovannini, G., Giroletti, M., \& Taylor, G. B. 2009 A $\& A$ 505, 509

Liuzzo, E., Giroletti, M., Giovannini, G., Boccardi, B., et al. 2013 A $\& A$ 560, 23

Massaro, E., Giommi, P., Leto, C., et al. 2009 A $\& A$ 495, 691

Rector, T. A., Gabuzda, D. C., \& Stocke, J. T. 2003 AJ 125, 1060

Wu, Z., Jiang, D. R., Gu, M., \& Liu, Y. 2007 A $\& A$ 466, 63 